Article

\title{
Uncertainty Relations: Curiosities and Inconsistencies
}

\section{Krzysztof Urbanowski}

Institute of Physics, University of Zielona Góra, ul. Prof. Z. Szafrana 4a, 65-516 Zielona Góra, Poland; K.Urbanowski@if.uz.zgora.pl or k.a.urbanowski@gmail.com

Received: 17 August 2020; Accepted: 22 September 2020; Published: 6 October 2020

\begin{abstract}
Analyzing general uncertainty relations one can find that there can exist such pairs of non-commuting observables $A$ and $B$ and such vectors that the lower bound for the product of standard deviations $\Delta A$ and $\Delta B$ calculated for these vectors is zero: $\Delta A \cdot \Delta B \geq 0$. Here we discuss examples of such cases and some other inconsistencies which can be found performing a rigorous analysis of the uncertainty relations in some special cases. As an illustration of such cases matrices $(2 \times 2)$ and $(3 \times 3)$ and the position-momentum uncertainty relation for a quantum particle in the box are considered. The status of the uncertainty relation in $\mathcal{P} \mathcal{T}$-symmetric quantum theory and the problems associated with it are also studied.
\end{abstract}

Keywords: uncertainty relations; uncertainties in $\mathcal{P} \mathcal{T}$-symmetric quantum mechanics

\section{Introduction}

The famous Heisenberg uncertainty relations [1,2] play an important and significant role in the understanding of the quantum world and in explanations of its properties. There is a mathematically rigorous derivation of the position-momentum uncertainty relation and the uncertainty relation for any pair of non-commuting observables, say $A$ and $B$, within the standard formalism of Schrödinger and von Neumann [3-6]. Among physicists who do not deal with theoretical research on the mathematical foundations of quantum mechanics, and in particular with a rigorous derivation of the uncertainty principles, there is an almost common belief based on the textbooks treatment of the problem (see e.g., $[7,8]$ ) that if one has a pair of non-commuting observables $A$ and $B$ then the the product of standard deviations $\Delta A$ and $\Delta B$ calculated for them is always large than some nonzero positive number, say $c$ :

$$
\Delta A \cdot \Delta B \geq c>0
$$

Here we show that such a belief may lead to confusions: It appears that there may exist such vectors that the lower bound for this product is zero. Simply, there exist such pairs of non-commuting operators $A$ and $B$ and such vectors from the Hilbert state space that for the standard deviations calculated for these vectors there is $\Delta A \cdot \Delta B \geq 0$ (see, e.g., [9]). The motivation of the paper is to examine such and similar cases and to discuss other limitations of Robertson-Schrödinger uncertainty relation (1) and inconsistencies as well as mathematical problems connected with this relation. Here we show examples of the cases where one can find that there is $\Delta A \cdot \Delta B \geq 0$ for some vectors although $[A, B] \neq 0$. The simplest cases are illustrated using Pauli matrices and Gell-Mann matrices. One meets a much more complicated situation in the case of a problem of a quantum particle in the box with perfectly reflecting and impenetrable walls: In this case we analyze the position-momentum uncertainty relation. We show that this problem leads to some paradoxical situations and generates some inconsistencies. The solution of these inconsistencies is proposed: From the point of view of the classical mechanics the particle in the box is a constrained system and the 
use of the position operator consistent with the constraints can solve these inconsistencies. Analyzing the problem of particle in the box we observed that some subtle properties of such system depending on the choice of the boundary conditions may be related to the symmetry properties of the problem under study. For this reason, we have attempted to investigate the problem of the uncertainty relations in $\mathcal{P} \mathcal{T}$-symmetric quantum mechanics. We found that within $\mathcal{P} \mathcal{T}$ symmetric quantum mechanics a relation corresponding to the uncertainty relations discussed, e.g., in [1-4], (or [5]), may not exist for every pair of non-commuting operators. We also found that if it exists for a pair of noncommuting $\mathcal{P} \mathcal{T}$-symmetric observables than it can not be considered as universally valid.

The paper is organized as follows: In Section 2 the reader finds some preliminaries. The case of Pauli and Gell-Mann matrices is analyzed in Section 3. Section 4 contains analysis of the case of a quantum particle in the box with perfectly reflecting and impenetrable walls. Discussion of the problem of uncertainty relations in $\mathcal{P} \mathcal{T}$-symmetric quantum theory is presented in Section 5 . Section 6 contains a discussion and conclusions.

\section{Preliminaries}

The uncertainty principle was formulated by Heisenberg [1,2] for the position and momentum and it can be written as follows

$$
\Delta_{\phi} X \cdot \Delta_{\phi} P_{x} \geq \frac{\hbar}{2} .
$$

Heisenberg considered $\Delta_{\phi} X$ and $\Delta_{\phi} P_{x}$ as "precisions" with which the values $x$ and $p$ are known [1]. Practically from the moment of the publication of Heisenberg's works [1,2], the ongoing discussion on how to interpret the inequality (2) began (see, e.g., [10-12]).

The contemporary interpretation of $\Delta_{\phi} X$ and $\Delta_{\phi} P_{x}$ considered in this paper comes from the derivation of the uncertainty relation made by Robertson [3] and Schrödinger [4,5], (see also [6]): $\Delta_{\phi} X$ and $\Delta_{\phi} P_{x}$ denote the standard (root-mean-square) deviations or variances. In a general case for an self-adjoint operator $F$ acting in $\mathcal{H}$ the standard deviation is defined as follows

$$
\Delta_{\phi} F=\| \delta F|\phi\rangle \|
$$

where $\delta F=\left(F-\langle F\rangle_{\phi} \mathbb{I}\right)$, and $\langle F\rangle_{\phi} \stackrel{\text { def }}{=}\langle\phi|F| \phi\rangle$ is the average (or expected) value of $F$ calculated for the normalized vector $|\phi\rangle \in \mathcal{H}$, provided that $|\langle\phi|F| \phi\rangle|\langle\infty$. (Note that from the definition of $\delta F$ it follows that $\delta F$ must be the self-adjoint operator if $F$ is self-adjoint). The equivalent definition is: $\Delta_{\phi} F \equiv \sqrt{\left\langle F^{2}\right\rangle_{\phi}-\langle F\rangle_{\phi}^{2}}$. (In Equation (2) $F$ denotes position and momentum operators $x$ and $p_{x}$ as well as their squares). Within the quantum theory the operator $F$ represents observable $F$. So, the uncertainty principle is a relation connecting standard deviations (variances) calculated for a pair of non-commuting observables (that is, self-adjoint operators) acting in a Hilbert space $\mathcal{H}$. In general, relations (1) and (2) results from basic assumptions of the quantum theory and from the geometry of Hilbert space [13]. Relations having the form (1) hold for any two observables, say $A$ and $B$, represented by non-commuting self-adjoint operators $A$ and $B$ acting in the Hilbert space of states (see [3] and also [4,5]), such that $[A, B]$ exists and $|\phi\rangle \in \mathcal{D}(A B) \cap \mathcal{D}(B A),(\mathcal{D}(\mathcal{O})$ denotes the domain of an operator $\mathcal{O}$ or of a product of operators):

$$
\Delta_{\phi} A \cdot \Delta_{\phi} B \geq \frac{1}{2}\left|\langle[A, B]\rangle_{\phi}\right|
$$

As it was said in the general case the relation (4) results from the geometry of the Hilbert space, strictly speaking from the Schwartz inequality: Let $\left|\psi_{1}\right\rangle,\left|\psi_{2}\right\rangle \in \mathcal{H}$, then one has $\left|\left\langle\psi_{1} \mid \psi_{2}\right\rangle\right| \leq$ $\|\left|\psi_{1}\right\rangle\|\|\left|\psi_{2}\right\rangle \|$. Next taking $\left|\psi_{1}\right\rangle=\delta A|\phi\rangle$ and $\left|\psi_{2}\right\rangle=\delta B|\phi\rangle$, after some algebra one obtains the inequality (4) - details can be found in Section 2 in [9] and in many textbooks and journal articles. Now if to identify operators $A$ and $B$ acting in the Hilbert space $\mathcal{H}=L^{2}(\mathbb{R}): A$ with 
the momentum operator, $P_{x}$, in quantum theory, $B$ with the position operator $X$, and then using the commutation relation,

$$
\left[P_{x}, X\right]=-i \hbar \mathbb{I},
$$

one obtains from (4) the inequality (2), i.e., the Heisenberg uncertainty relation.

Note that starting with the Schwartz inequality all subsequent calculations and transformations leading to the result (4) are purely mathematical operations and there is no physics in them (see, e.g., [9]): The inequality (4) is a purely mathematical inequality and examining when and for which vectors it occurs and for which it does not occur is a mathematical task. Physics will appear only when physical quantities are assigned to operators $A$ and $B$ and the Hilbert space on which they act is identified with the space of the states of the physical system considered.

As it was mentioned, there is still a discussion on how to interpret inequalities (4) and (2) and how to improve them (see, e.g., [10] and references therein, [11,12,14-20] and many other papers). From the derivation of the formula (4) it follows that the standard deviations $\Delta_{\phi} A$ and $\Delta_{\phi} B$ characterize the statistical distribution of the most probable values of $A$ and $B$ in the state $|\phi\rangle$. The inequality (4) does not depend on a possible influence of the measuring device on the result of measurements and on the statistical distribution of values of $A$ and $B$ measured by this device. So, it seems that a safe interpretation of (4) is the interpretation close to that one can find in [21], namely that it is impossible to prepare a system in a state $|\phi\rangle$ that non-commuting observables $A$ and $B$ have both their probability distributions of values of $A$ and $B$ in this state sharply concentrated around a single value (see, [10-12]). Therefore the relation (4) is sometimes called the "preparation uncertainty relation" $[10-12,18,19]$. There is also another, probably the most popular interpretation of inequality (2) in the literature. Namely, Heisenberg's relation (2) is considered as a trade-off between the precision $\Delta_{\phi} X$ of an approximate position measurement and the momentum disturbance $\Delta_{\phi} P$ incurred by that measurement (see, e.g., [10,19]). This is the "error-disturbance" or "noise-disturbance uncertainty relation" (see, e.g., [19,22-26]. The relation (4) can be understood analogously. The proof of this relation having similar form to the relations (2), (4) can be found, e.g., in [19,22,24]. One more interpretation of the uncertainty relation can be found in the literature. It is so-called Heisenberg "uncertainty relation for joint measurements". It can be generally formulated as follows [22]: For any apparatus $\mathbb{A}$ with two outputs for the joint measurement of $A$ and $B$, the relation (4) holds for any input state $|\phi\rangle$, where in this case $\Delta_{\phi} A$ is replaced by $\epsilon(A, \phi, \mathbb{A}), \Delta_{\phi} B \rightarrow \epsilon(B, \phi, \mathbb{A})$ and $\epsilon(X, \phi, \mathbb{A})$ stands for the noise of the $X$ measurement in state $|\phi\rangle$ using apparatus $\mathbb{A}$ for $X=A, B$ [22,26-28]. The proof of this relation can be found, e.g., in [28]. It requires the assumption that the experimental mean values of the outcome $x_{A}$ of the $A$ measurement and the outcome $y_{B}$ of the $B$ measurement should coincide with the mathematical expectation values of observables $A$ and $B$, respectively, on any input state $|\phi\rangle$ [28]. So, due to such an assumption the final form of the uncertainty relation for joint measurements is analogous to that given by the inequality (4). In general, a common feature of all these cases is that the uncertainty relation takes the form considered in this paper, that is the form given by inequalities (1), (2) and (4). A discussion of different aspects of these interpretations as well as attempts to improve uncertainty relations are still continued and can be found in many papers (see, e.g., [29-33]).

In this paper attention will be focused on the definition of standard deviations given by the formula (3) and properties of (4) resulting from this definition. It has been pointed out in [9] that it is not necessary for $A$ and $B$ to commute, $[A, B]=0$, in order that $\langle\phi|[A, B]| \phi\rangle=0$ for some $|\phi\rangle \in \mathcal{H}$. Simply it may happen that for some $|\phi\rangle \in \mathcal{H}$ and for some non-commuting operators $A$ and $B$ the expectation value of the commutator $[A, B]$ vanishes: $\langle\phi|[A, B]| \phi\rangle=0$ and then the inequality (4) takes the following form:

$$
\Delta_{\phi} A \cdot \Delta_{\phi} B \geq 0 \text {. }
$$

This means that in such cases the inequality (4) having the form (6) does not impose any restrictions for the values of $\Delta_{\phi} A$ and $\Delta_{\phi} B$ besides the condition that there should be $0 \leq \Delta_{\phi} A<\infty$ and $0 \leq \Delta_{\phi} B<\infty$. Examples of such and similar cases will be analyzed in the next Section. 


\section{Simple Algebraic Examples}

Here we present examples of self-adjoint operators (matrices) for which the inequality (4) has the form (6). So, let us considerer for a start the simplest case of $(2 \times 2)$ matrices. Using Pauli matrices

$$
\sigma_{x}=\left(\begin{array}{cc}
0 & 1 \\
1 & 0
\end{array}\right), \sigma_{y}=\left(\begin{array}{cc}
0 & -i \\
i & 0
\end{array}\right), \sigma_{z}=\left(\begin{array}{cc}
1 & 0 \\
0 & -1
\end{array}\right)
$$

one has $\sigma_{x}=\sigma_{x}^{+}, \sigma_{y}=\sigma_{y}^{+}, \sigma_{z}=\sigma_{z}^{+}$and

$$
\left[\sigma_{x}, \sigma_{y}\right]=2 i \sigma_{z}
$$

Identifying $\sigma_{x}, \sigma_{y}$ with operators $A$ and $B$ respectively one can rewrite (4) as follows

$$
\Delta_{\phi} \sigma_{x} \cdot \Delta_{\phi} \sigma_{y} \geq \frac{1}{2}\left|\left\langle\left[\sigma_{x}, \sigma_{y}\right]\right\rangle_{\phi}\right| \equiv\left|\left\langle\sigma_{z}\right\rangle_{\phi}\right|
$$

where

$$
|\phi\rangle=N\left(\begin{array}{l}
a \\
b
\end{array}\right),
$$

$N=\left(|a|^{2}+|b|^{2}\right)^{-1 / 2}, a, b \in \mathbb{C},\left\langle\sigma_{x}\right\rangle_{\phi}=\left\langle\phi\left|\sigma_{x}\right| \phi\right\rangle=2 N^{2} \Re\left[a^{*} b\right]$ and $\left(\Delta_{\phi} \sigma_{x}\right)^{2}=\left\langle\phi\left|\sigma_{x}^{2}\right| \phi\right\rangle-\left\langle\sigma_{x}\right\rangle_{\phi}^{2} \equiv$ $1-4 N^{4}\left(\Re\left[a^{*} b\right]\right)^{2}$, and so on. (Here $\Re[z]$ and $\Im[z]$ denote real and imaginary parts of $z$ respectively). It is easy to see that $\left\langle\sigma_{z}\right\rangle_{\phi}=\left\langle\phi\left|\sigma_{z}\right| \phi\right\rangle=N^{2}\left(|a|^{2}-|b|^{2}\right)$ which means that $\left|\left\langle\sigma_{z}\right\rangle_{\phi}\right|>0$ if $|a| \neq|b|$. Choosing $|\phi\rangle$ such that $|a|=|b|$, e.g., $a=b=1$,

$$
|\phi\rangle \Rightarrow\left|\phi_{1}\right\rangle=\frac{1}{\sqrt{2}}\left(\begin{array}{l}
1 \\
1
\end{array}\right)
$$

one finds that $\left|\left\langle\sigma_{z}\right\rangle_{\phi_{1}}\right| \equiv 0$, and, as a result the inequality (9) will take the form of (6) for $|\phi\rangle_{1}$. We have $\left\langle\sigma_{y}\right\rangle_{\phi}=2 N^{2} \Im\left[a^{*} b\right]$ and $\Delta_{\phi} \sigma_{y}=1-4 N^{4}\left(\Im\left[a^{*} b\right]\right)^{2}$. This means that for $\left|\phi_{1}\right\rangle$ one obtains $\left\langle\sigma_{y}\right\rangle_{\phi_{1}}=0$ and $\Delta_{\phi_{1}} \sigma_{y}=1$. Note that in this case $\Delta_{\phi_{1}} \sigma_{x}=0$ because the vector $\left|\phi_{1}\right\rangle$ is an eigenvector of $\sigma_{x}$, which means that the both sides of the inequality (9) are equal to zero for $|\phi\rangle=\left|\phi_{1}\right\rangle$ as it should be in such a case.

A little more complicated example can be found considering $(3 \times 3)$ matrices. So, let us consider Gell-Mann matrices $\lambda_{3}, \lambda_{4}$ and $\lambda_{5}$ as an example:

$$
\lambda_{3}=\left(\begin{array}{ccc}
1 & 0 & 0 \\
0 & -1 & 0 \\
0 & 0 & 0
\end{array}\right), \lambda_{4}=\left(\begin{array}{lll}
0 & 0 & 1 \\
0 & 0 & 0 \\
1 & 0 & 0
\end{array}\right), \lambda_{5}=\left(\begin{array}{ccc}
0 & 0 & i \\
0 & 0 & 0 \\
-i & 0 & 0
\end{array}\right) .
$$

They are self-adjoint and do not commute,

$$
\left[\lambda_{3}, \lambda_{4}\right]=-i \lambda_{5} \neq 0
$$

For these matrices the inequality (4) takes the following form,

$$
\Delta_{\psi} \lambda_{3} \cdot \Delta_{\psi} \lambda_{4} \geq \frac{1}{2}\left|\left\langle\left[\lambda_{3}, \lambda_{4}\right]\right\rangle_{\psi}\right| \equiv \frac{1}{2}\left|\left\langle\lambda_{5}\right\rangle_{\psi}\right|,
$$

where

$$
|\psi\rangle=\frac{1}{\sqrt{|a|^{2}+|b|^{2}+|c|^{2}}}\left(\begin{array}{l}
a \\
b \\
c
\end{array}\right)
$$


and $a, b, c \in \mathbb{C}$. Now, putting $a=b=c=1$ in (15) one gets

$$
|\psi\rangle \Rightarrow\left|\psi_{1}\right\rangle=\frac{1}{\sqrt{3}}\left(\begin{array}{l}
1 \\
1 \\
1
\end{array}\right)
$$

which leads to the result $\left|\left\langle\lambda_{5}\right\rangle_{\psi_{1}}\right|=0$, and hence one concludes that for $\left|\psi_{1}\right\rangle$ the inequality (14) takes the following form

$$
\Delta_{\psi_{1}} \lambda_{3} \cdot \Delta_{\psi_{1}} \lambda_{4} \geq 0
$$

exactly as the inequality (6). More detailed analysis leads to the surprising result: If in (15) $a=$ $a^{*}, b=b^{*}, c=c^{*}$ then there is $\left|\left\langle\lambda_{5}\right\rangle_{\psi}\right|=0$ for any such $a, b, c$. Hence for $|\psi\rangle$ defined by real $a, b, c$ the uncertainty relation (14) takes the same form as the relation (6). On the other hand if to consider the more general case when $a, b, c$ are the complex numbers then only for

$$
c=\beta a,
$$

(where $\beta=\beta^{*} \neq 0$ ), one obtains that $\left|\left\langle\lambda_{5}\right\rangle_{\psi}\right|=0$ for any $a$ and $b$ but $\left|\left\langle\lambda_{5}\right\rangle_{\psi}\right|>0$ for these $a$ and $c$, which do not fulfil the condition (18) and in this case the uncertainty relation (14) has the standard form. Similar examples can be found for self-adjoint matrices or operators acting in any Hilbert space (see, e.g., Section 2 in [20]).

\section{Particle in the Box}

Many similar situations to those discussed in the previous Section, or even paradoxes, can be found when one is analyzing properties of a quantum particle, which spatial motion is confined to a finite volume. Usually such cases are much more complicated than that discussed in the previous Section. As a simplest nontrivial example of such a case the problem of a quantum particle in the box with perfectly reflecting and impenetrable (rigid) walls will be considered in this Section. We assume that a quantum non-relativistic particle of mass $m$ is mowing on an interval $(a, b)$ of the real axis. In other words we assume that this particle is in the potential well $V(x)$ defined as follows

$$
V(x)=\left\{\begin{array}{cl}
0 & \text { for } a<x<b \\
+\infty & \text { for } x \leq a \text { and } x \geq b
\end{array}\right.
$$

The Hamiltonian $H$, of such a system has a usual form: It is the sum of the kinetic energy, $T$, and the potential $V(x)$ : That is $H=T+V(x)$. The assumed potential $V(x)$ forces the particle to be somewhere between $a$ and $b$. Hence in the position representation the probability $|\psi(x)|^{2} d x$, (where $\psi(x)=|\psi(x)\rangle$ is the wave function of the particle), to find this particle having position between $x$ and $x+d x$ out of the interval $(a, b)$ must be zero. Therefore it must be $|\psi(x)|^{2}=0$ for $x<a$ and $x>b$, and thus within this problem there must be

$$
\psi(x)=0 \text { for } x<a \text { and } x>b .
$$

Taking into account that in this paper we analyze some properties of the uncertainty relation our attention will be focussed only on the operator corresponding to the momentum of the particle considered. In one dimensional models on the real line the position operator $X$ and the momentum operator $P_{x}$ are a self-adjoint operators and when they act in the Hilbert space $\mathcal{H}=L^{2}(\mathbb{R})$, (where $L^{2}(\mathbb{R})$ denotes the space of square integrable functions on the real line $\mathbb{R}$ ), they are defined by $X \psi(x)=x \psi(x),($ or $X|\psi(x)\rangle=x|\psi(x)\rangle), P_{x} \phi(x)=-i \hbar \frac{d}{d x} \phi(x),\left(\right.$ or $\left.P_{x}|\phi(x)\rangle=-i \hbar \frac{d}{d x}|\phi(x)\rangle\right)$ to act on appropriate sets of functions $|\psi(x)\rangle,|\phi(x)\rangle \in L^{2}(\mathbb{R})$. Now if the motion of the particle is confined to a segment $[a, b] \subset \mathbb{R}$, then the support of the corresponding wave-functions is $[a, b]$ and thus they form a subspace of $L^{2}(\mathbb{R})$, which is identified with the Hilbert space of square integrable functions $L^{2}([a, b])$ 
on $[a, b]$. The problem is that there is no a self-adjoint operator acting as $-i \hbar \frac{d}{d x}$ in the subspace of square integrable functions in $L^{2}([a, b])$ defined by the condition (20), that is, which vanish at the endpoints of the interval $[a, b]$.

Let us pass now to the analysis of properties of an operator corresponding to the momentum of the particle considered. For simplicity we will consider the "standard" case when $a=0$ and $b=l>0$ and the "symmetric" case when $a=-\frac{l}{2}$ and $b=+\frac{l}{2}$, (see, e.g., [34]).

\subsection{The "Standard" Case}

Let us consider now the operator $P_{x}$ in a closed interval $[0, l] \ni x$ and let us take for a domain $D\left(P_{x}\right)$ the following subspace of $L^{2}([0, l])$,

$$
D\left(P_{x}\right)=\left\{\phi(x), \phi^{\prime}(x) \in L^{2}([0, l]): \phi(0)=\phi(l)=0\right\}
$$

where $\phi^{\prime}(x)=\frac{d}{d x} \phi(x)$. It appears that such defined $P_{x}$ is only a symmetric operator in $D\left(P_{x}\right)$ but it is not a self-adjoint in $D\left(P_{x}\right)$, (see, e.g., [35-38] and references therein). If one needs a self-adjoint extension of $P_{x}$ then one have to change boundaries defining $D\left(P_{x}\right)$. There is a family of self-adjoint extensions of $P_{x}$ "numbered" by a real parameter $\vartheta$, where $0 \leq \vartheta<2 \pi$ [35-38], which are denoted as $P_{x}^{\vartheta}$ :

$$
\begin{gathered}
P_{x}^{\vartheta} \phi(x)=-i \hbar \frac{d}{d x} \phi(x), \\
D\left(P_{x}^{\vartheta}\right)=\left\{\phi(x), \phi^{\prime}(x) \in L^{2}([0, l]): \phi(l)=e^{i \vartheta} \phi(0)\right\} .
\end{gathered}
$$

Note that the set being the domain $D\left(P_{x}^{\vartheta}\right)$ of the operator $P_{x}^{\vartheta}$ is much larger than the set defined in (21): Functions belonging to $D\left(P_{x}^{\vartheta}\right)$ do not have to meet the condition $\phi(0)=0$. This definition leads to the following solutions of the eigenvalue problem for $P_{x}^{\vartheta}$ : One finds that the eigenfunctions are

$$
u_{n}^{\vartheta}(x)=\frac{1}{\sqrt{l}} e^{\frac{i}{\hbar} p_{n}^{\vartheta} x}
$$

where $n=0, \pm 1, \pm 2, \ldots$ and the corresponding eigenvalues are:

$$
p_{n}^{\vartheta}=\hbar \frac{2 \pi n+\vartheta}{l} .
$$

For each $\vartheta$ the eigenfunctions $u_{n}^{\vartheta}(x)$ form an orthonormal basis in $L^{2}([0, l])$. Let us analyze now the uncertainty relation (4) for the operators $X$ and $P_{x}^{\vartheta}$. For each $u_{n}^{\vartheta}(x)=\left|u_{n}^{\vartheta}(x)\right\rangle$ there is $\Delta_{u_{n}^{\vartheta}} X<l$ and $\Delta_{u_{n}^{\vartheta}} P_{x}^{\vartheta}=0$. From this one concludes that there is

$$
\Delta_{u_{n}^{\vartheta}} X \cdot \Delta_{u_{n}^{\vartheta}} P_{x}^{\vartheta}=0
$$

which contradicts (2) and (5). This result suggests that in the case considered there is something wrong with the commutation relation (5) and with the modulus of the expectation value of $\left\langle u_{n}^{\vartheta}(x)\left|\left[P_{x}^{\vartheta}, X\right]\right| u_{n}^{\vartheta}(x)\right\rangle$. There is

$$
\left\langle u_{n}^{\vartheta}(x)\left|\left[P_{x}^{\vartheta}, X\right]\right| u_{n}^{\vartheta}(x)\right\rangle=\left\langle u_{n}^{\vartheta}(x)\left|P_{x}^{\vartheta} X\right| u_{n}^{\vartheta}(x)\right\rangle-\left\langle u_{n}^{\vartheta}(x)\left|X P_{x}^{\vartheta}\right| u_{n}^{\vartheta}(x)\right\rangle,
$$

and more detailed analysis shows that the position operator $X$ removes vectors $|\phi(x)\rangle \in D\left(P_{x}^{\vartheta}\right)$ from the domain $D\left(P_{x}^{\vartheta}\right)$ of $P_{x}^{\vartheta}$. Simply, there is $X|\phi(x)\rangle=x|\phi(x)\rangle \stackrel{\text { def }}{=}|\chi(x)\rangle$ and, as one can see, the condition $\chi(l)=e^{i \vartheta} \chi(0)$ guaranteing that $\chi(x) \in D\left(P_{x}^{\vartheta}\right)$ can not be fulfilled for such $|\chi(x)\rangle$. This means that the commutator $\left[P_{x}^{\vartheta}, X\right]$ does not exist in the considered case (see [37]). This conclusion concerns also eigenvectors $u_{n}^{\vartheta}(x)$ of $P_{x}^{\vartheta}$ : The position operator $X$ also removes vectors 
$u_{n}^{\vartheta}(x)$ from the domain $D\left(P_{x}^{\vartheta}\right)$. For every $\chi_{n}^{\vartheta}(x) \stackrel{\text { def }}{=} X u_{n}^{\vartheta}(x) \equiv x u_{n}^{\vartheta}(x)$ one finds that $\chi_{n}^{\vartheta}(l) \equiv l u_{n}^{\vartheta}(l) \neq 0$, whereas $\chi_{n}^{\vartheta}(0) \equiv 0 \cdot u_{n}^{\vartheta}(0)=0$ which means that $\chi_{n}^{\vartheta}(x)=x u_{n}^{\vartheta}(x)$ does not belong to the domain $D\left(P_{x}^{\vartheta}\right)$ and therefore the matrix element $\left\langle u_{n}^{\vartheta}(x)\left|P_{x}^{\vartheta} X\right| u_{n}^{\vartheta}(x)\right\rangle$ is not defined. Hence the relation (27) is not defined. This analysis shows that in the considered "standard" case of the particle, which motion is confined to a segment $[0, l]$, the uncertainty relation (2) does not hold [37].

\subsection{The "Symmetric" Case}

Let us now analyze the symmetric" case of the particle in the box when the particle can move only inside the segment $\left[-\frac{l}{2}, \frac{l}{2}\right]$. In this case

$$
V(x)=V^{*}(x)=\left\{\begin{array}{cc}
0 & \text { for }|x| \leq \frac{l}{2}, \\
+\infty & \text { for }|x|>\frac{1}{2} .
\end{array},\right.
$$

the family of self-adjoint extensions $\Pi_{x}^{\vartheta}$ of the operator $P_{x}$ is defined as follows [39]:

$$
\begin{gathered}
\Pi_{x}^{\vartheta} \phi(x)=-i \hbar \frac{d}{d x} \phi(x), \\
D\left(\Pi_{x}^{\vartheta}\right)=\left\{\phi(x), \phi^{\prime}(x) \in L^{2}([0, l]): \phi\left(\frac{l}{2}\right)=e^{i \vartheta} \phi\left(-\frac{l}{2}\right)\right\},
\end{gathered}
$$

and again $0 \leq \vartheta<2 \pi, n=0, \pm 1, \pm 2, \ldots$. The solutions of the eigenvalue problem for $\Pi_{x}^{\vartheta}$ have the same form as for the operator $P_{x}^{\vartheta}$ : eigenfunctions are given by (24) and eigenvalues $p_{n}^{\vartheta}$ are given by the formula (25). Considering the uncertainty relations (2) and (4) for $X$ and $\Pi_{x}^{\vartheta}$ computed for $|\phi\rangle=\left|u_{n}^{\vartheta}(x)\right\rangle$ one finds again that $\Delta_{u_{n}^{\vartheta}} X<l$ and $\Delta_{u_{n}^{\vartheta}} \Pi_{x}^{\vartheta}=0$, which suggest that in the considered case there is $\Delta_{u_{n}^{\vartheta}} X \cdot \Delta_{u_{n}^{\vartheta}} \Pi_{x}^{\vartheta}=0$ too, which again contradicts (2). Now if one wants to verify this conclusion one should use the relation (4), and then one should to compute the expectation value of $\left\langle u_{n}^{\vartheta}(x)\left|\left[\Pi_{x}^{\vartheta}, X\right]\right| u_{n}^{\vartheta}(x)\right\rangle$. The properties of the matrix element $\left\langle u_{n}^{\vartheta}(x)\left|\Pi_{x}^{\vartheta} X\right| u_{n}^{\vartheta}(x)\right\rangle$ were the crucial in the previously considered "standard" case. So, let us analyze the function $\xi_{n}^{\vartheta}(x) \stackrel{\text { def }}{=} X\left|u_{n}^{\vartheta}(x)\right\rangle$ and let us check if (and when) $\xi_{n}^{\vartheta}(x) \in D\left(\Pi_{x}^{\vartheta}\right)$. There are

$$
\xi_{n}^{\vartheta}\left(\frac{l}{2}\right)=\frac{l}{2} u_{n}^{\vartheta}\left(\frac{l}{2}\right) \text { and } \xi_{n}^{\vartheta}\left(-\frac{l}{2}\right)=-\frac{l}{2} u_{n}^{\vartheta}\left(-\frac{l}{2}\right) .
$$

Thus boundaries $\xi_{n}^{\vartheta}\left(\frac{l}{2}\right)=e^{i \vartheta} \xi_{n}^{\vartheta}\left(-\frac{l}{2}\right)$ (see (30)) and properties (31) lead to the following conclusion: In the "symmetric" case $\xi_{n}^{\alpha}(x)=x u_{n}^{\alpha}(x) \in D\left(\Pi_{x}^{\alpha+\pi}\right)$ for all $\alpha$, such that $0<\alpha<\pi$. It is because $(-1)$ can be represented by $e^{i \pi} \equiv-1$. In other words there exists a subfamily of self-adjoint extension of $\Pi_{x}^{\alpha}$, where $0<\alpha<\pi$, such $\xi_{n}^{\alpha}(x)=x u_{n}^{\alpha}(x) \in D\left(\Pi_{x}^{\alpha+\pi}\right)$, and in general $X D\left(\Pi_{x}^{\alpha}\right) \rightarrow$ $D\left(\Pi_{x}^{\alpha+\pi}\right) \neq D\left(\Pi_{x}^{\alpha}\right)$. So, for $0<\alpha<\pi$ position operator $X$ moves eigenfunctions of $\Pi_{x}^{\alpha}$ from $D\left(\Pi_{x}^{\alpha}\right)$ to domain of $\Pi_{x}^{\alpha+\pi}$ but nevertheless $X u_{n}^{\alpha}(x) \equiv x u_{n}^{\alpha}(x) \notin D\left(\Pi_{x}^{\alpha}\right)$ again. For $\pi \leq \vartheta<2 \pi$ eigenfunctions of $\Pi_{x}^{\vartheta}$ are removed from any domain of the family of self-adjoint extensions $\Pi_{x}^{\vartheta}$ of the operator $-i \hbar \frac{d}{d x}$. It is easy to show that $\left\langle\phi(x)\left|\Pi_{x}^{\alpha} X\right| \phi(x)\right\rangle \equiv\left\langle\phi(x) \mid \Pi_{x}^{\alpha}(X \phi(x))\right\rangle \neq\left\langle\left(\Pi_{x}^{\alpha} \phi(x)\right) \mid(X \mid \phi(x))\right\rangle$ for $|\phi(x)\rangle \in D\left(\Pi_{x}^{\alpha}\right)$. This property leads to a rather unexpected result that $\left\langle u_{n}^{\alpha}(x)\left|\left[\Pi_{x}^{\alpha}, X\right]\right| u_{n}^{\alpha}(x)\right\rangle$ does not exist not only for every $0<\alpha<\pi$ but also for any $\vartheta$, such that $0<\vartheta<2 \pi$. Note that if $|\phi(x)\rangle=\left|u_{n}^{\alpha}\right\rangle$ then, contrary to the above conclusion, one expects that $\left\langle u_{n}^{\alpha}(x)\left|\left[\Pi_{x}^{\alpha}, X\right]\right| u_{n}^{\alpha}(x)\right\rangle=0$. Ignoring the above described subtleties one can see that in the "symmetric" case the situation is the same as in the "standard" case. Again the left hand side of the inequality (4) computed for $X$ and $\Pi_{x}^{\vartheta}$ and $|\phi\rangle=\left|u_{n}^{\vartheta}(x)\right\rangle$ takes the zero value, $\Delta_{u_{n}^{\vartheta}} X \cdot \Delta_{u_{n}^{\vartheta}} \Pi_{x}^{\vartheta}=0$, and the right hand side of (4) does not exist. In [39] a conclusion was that in such a case the momentum is not a physical observable and therefore a consideration of such a case has no a physical justification. As it was said earlier, we analyze properties of uncertainty relations considering them as a mathematical problem and we are interested in finding 
mathematical solutions of this problem. It seems that a solution to a "paradox" such "paradoxes can be found by carrying out a more detailed analysis of the case under considerations.

From the point of view of the theoretical mechanics the system considered is the constrained system. Simply here imposed on the positions of the considered particle are restrictions of the geometrical nature, called constraints. In such a situation the constraint means that certain positions of the particle are permissible and others are forbidden: In the case considered the allowed position, $x$, are: $-\frac{l}{2} \leq x \leq \frac{l}{2}$, whereas $x<-\frac{l}{2}$ and $x>\frac{l}{2}$ are the forbidden positions. The equation of these constraints can be written as follows:

$$
|x| \leq \frac{l}{2}
$$

It seems that a possible solution to the problem may be choosing the position operator $X$ in such a way that it would be consistent with constraints. So, taking into account the constraint equation one can define modified position operator $X_{M}$ acting in $L^{2}(\mathbb{R})$ as follows

$$
\begin{gathered}
X_{M}|\psi(x)\rangle=x \Theta\left(\frac{l}{2}+x\right) \Theta\left(\frac{l}{2}-x\right)|\psi(x)\rangle, \\
D\left(X_{M}\right)=\left\{\psi(x) \in L^{2}(\mathbb{R}): \psi(x)=0 \text { for }|x|>\frac{l}{2}\right\},
\end{gathered}
$$

where $\Theta(x)$ is the unit step function: $\Theta(x)=1$ for $x \geq 0$ and $\Theta(x)=0$ for $x<0$. Note that all functions $\phi(x)$ having the set $x \in\left[-\frac{l}{2}, \frac{l}{2}\right]$ as a support and belonging to $L^{2}\left(\left[-\frac{l}{2}, \frac{l}{2}\right]\right)$ belong also to the domain $D\left(X_{M}\right)$. Using the modified position operator $X_{M}$ one finds for $|\psi(x)\rangle \in L^{2}(\mathbb{R})$ that formally,

$$
\begin{aligned}
{\left[P_{x}, X_{M}\right]|\psi(x)\rangle=} & i \hbar \frac{l}{2}\left[\delta\left(\frac{l}{2}+x\right)+\delta\left(\frac{l}{2}-x\right)\right]|\psi(x)\rangle \\
& -i \hbar \Theta\left(\frac{l}{2}+x\right) \Theta\left(\frac{l}{2}-x\right)|\psi(x)\rangle .
\end{aligned}
$$

Note that here operators $P_{x}$ and $X_{M}$ act in $L^{2}(\mathbb{R})$. If a segment $\left[-\frac{l}{2}, \frac{l}{2}\right]$ is the support of $|\phi(x)\rangle$ then $|\phi(x)\rangle \in L^{2}\left(\left[-\frac{l}{2}, \frac{l}{2}\right]\right)$ and $\Theta\left(\frac{l}{2}+x\right) \Theta\left(\frac{l}{2}-x\right)|\phi(x)\rangle=|\phi(x)\rangle$, which implies that

$$
\left[P_{x}, X_{M}\right]|\phi(x)\rangle=i \hbar \frac{l}{2}\left[\delta\left(\frac{l}{2}+x\right)+\delta\left(\frac{l}{2}-x\right)\right]|\phi(x)\rangle-i \hbar|\phi(x)\rangle \text {. }
$$

Hence for normalized $|\phi(x)\rangle \in L^{2}\left(\left[-\frac{l}{2}, \frac{l}{2}\right]\right)$ one obtains that

$$
\left\langle\phi(x)\left|\left[P_{x}, X_{M}\right]\right| \phi(x)\right\rangle=i \hbar \frac{l}{2}\left[\left.|| \phi\left(-\frac{l}{2}\right)\right|^{2}+\left|\phi\left(\frac{l}{2}\right)\right|^{2}\right]-i \hbar .
$$

The right hand side of (37) is zero if

$$
\left|\phi\left(-\frac{l}{2}\right)\right|=\left|\phi\left(+\frac{l}{2}\right)\right|=\frac{1}{\sqrt{l}}
$$

In addition, the right hand side of (37) is zero for every $\phi(x) \in L^{2}\left(\left[-\frac{l}{2}, \frac{l}{2}\right]\right)$ such that $|\phi(x)|=\frac{1}{\sqrt{l}}$. Note that, among others, all eigenfunctions $u_{n}^{\vartheta}(x)$ (see (24)) of self-adjoint extensions of the momentum operator $P_{x}$ have these properties. On the other, if

$$
\left|\phi\left(-\frac{l}{2}\right)\right|=\left|\phi\left(+\frac{l}{2}\right)\right| \neq \frac{1}{\sqrt{l}} \text {, or, }\left|\phi\left(-\frac{l}{2}\right)\right| \neq\left|\phi\left(+\frac{l}{2}\right)\right|,
$$

then the right hand side of (37) is nonzero. 
Coming back to the uncertainty relations for the modified position operator $X_{M}$ and momentum $P_{x}$ one finds that

$$
\Delta_{\phi} X_{M} \cdot \Delta_{\phi} P_{x} \geq \hbar\left|\frac{l}{2}\left[\left|\phi\left(-\frac{l}{2}\right)\right|^{2}+\left|\phi\left(-\frac{l}{2}\right)\right|^{2}\right]-1\right| .
$$

So, it seems that the relation (40) is consistent with the uncertainty relation (4) and the use of the modified position operator may remove inconsistences with the position-momentum uncertainty relation for particle in the box. Note that, as it was mentioned, the right hand side of the inequality (40) can be zero for vectors satisfying conditions specified after Equation (37).

\section{Uncertainty Principles and $\mathcal{P} \mathcal{T}$-Symmetric Quantum Theory}

The uncertainty principle is one of the most famous predictions of quantum mechanics. As it was stated in [40] (see also [41]) "as deduced from within the quantum theory itself, the uncertainty principle only prohibits the possibility of preparing an ensemble of systems in which all those properties are sharply defined". This general statement can be translated for the case of two non-commuting observables $A$ and $B$ as follows: The possibility of preparing a system, in which the values of observables $A$ and $B$ are sharply defined, can not be realized. This is true within the Schrödinger and von Neumann quantum mechanics. The question is: Is this also true within the $\mathcal{P} \mathcal{T}$-symmetric quantum mechanics? Simply, when one goes form the standard (Schrödinger and von Neumann) quantum mechanics to $\mathcal{P} \mathcal{T}$-symmetric quantum mechanics one meets some surprises. One of them is the problem of the uncertainty relations. In standard quantum mechanics one can ask about exact values of the position and momentum of the particle independently of that if the Hamiltonian $H$ is known or not and independently of the form of $H$. It is because all observables act in the same, common Hilbert space $\mathcal{H}$ of states and the scalar product in $\mathcal{H}$ does not depend on the choice of the Hamiltonian $H$. The different situation is in $\mathcal{P} \mathcal{T}$-symmetric quantum mechanics, where the Hamiltonian $H$ and solutions of the eigenvalue problem for this $H$ determine the space of states and the "scalar product" in this space [42].

Within the $\mathcal{P} \mathcal{T}$-quantum mechanics the property that non-self-adjoint but $\mathcal{P} \mathcal{T}$-symmetric Hamiltonians can have the real eigenvalues is used. Here the $\mathcal{P} \mathcal{T}$-symmetric Hamiltonian means that the Hamiltonian $H$ is requested to satisfy the following condition,

$$
H^{P T} \stackrel{\text { def }}{=} \mathcal{P} \mathcal{T} H \mathcal{P} \mathcal{T} \equiv H
$$

where the operators $\mathcal{P}$ and $\mathcal{T}$ are defined as follows:

$$
\mathcal{P} x=-x, \mathcal{P} p_{x}=-p_{x}, \mathcal{T} x=x, \mathcal{T} p_{x}=-p_{x},
$$

and $x$ and $p_{x}$ denote position and momentum respectively,

$$
\mathcal{P} \phi(x)=\phi(-x), \quad \mathcal{T} \phi(x)=\phi^{*}(x) .
$$

When $\mathcal{T}$ acts in the Hilbert space or in a space with sesquilinear form, then $\langle\mathcal{T} \psi \mid \mathcal{T} \phi\rangle=\langle\phi \mid \psi\rangle$. From these definitions it follows that $\mathcal{P}^{2}=\mathcal{T}^{2}=\mathbb{I},[\mathcal{P}, \mathcal{T}]=0$ and that $(\mathcal{P} \mathcal{T})^{2}=\mathbb{I}$. This means that $\mathcal{P}=\mathcal{P}^{-1}, \mathcal{T}=\mathcal{T}^{-1}$ and $\mathcal{P} \mathcal{T}=(\mathcal{P} \mathcal{T})^{-1}$. Thus $\mathcal{P} X \mathcal{P}=-X, \quad \mathcal{P} P_{x} \mathcal{P}=-P_{x}, \mathcal{T} X \mathcal{T}=X, \mathcal{T} P_{x} \mathcal{T}=$ $-P_{x}$, where $X$ and $P_{x}$ are the standard position and momentum operators. In analogy to Hermitian quantum mechanics one can define the inner product in this case as

$$
(\psi, \phi)^{P T}=\int_{-\infty}^{+\infty}[\mathcal{P} \mathcal{T} \psi(x)] \phi(x) d x \equiv \int_{-\infty}^{+\infty} \psi^{*}(-x) \phi(x) d x,
$$

but, unfortunately, then one runs into the problem of having negative norm for some states. This problem can be solved by introducing a new operator usually called the $\mathcal{C}$ operator expressing a 
symmetry between the positive and negative norm states. Using this $\mathcal{C}$ operator we can define the $\mathcal{C P} \mathcal{T}$ inner product as follows

$$
(\psi, \phi)^{C P T}=\int \psi^{C P T}(x) \phi(x) d x
$$

where $\psi^{C P T}(x)=\mathcal{C}[\mathcal{P} \mathcal{T} \psi(x)]=\int \mathcal{C}(x, y) \psi^{*}(-y) d y$. This inner product satisfies the requirements for quantum theory defined by $H$ and the norm defined by means of this product is positive. In order to find within the $\mathcal{P} \mathcal{T}$-symmetric quantum mechanics a proper space of states with the proper inner product such as $\mathcal{C P} \mathcal{T}$ inner product (that is the $\mathcal{C}$ operator) one must find solutions of the eigenvalue problem,

$$
H \phi_{n}(x)=E_{n} \phi_{n}(x),
$$

for a given $\mathcal{P} \mathcal{T}$-symmetric Hamiltonian $H^{P T}$. If $H^{P T}=H$ then the eigenvalues $E_{n}$ are real. Having solutions of Equation (46) one can construct a suitable $\mathcal{C}$ operator, e.g., as follows [42-45]

$$
\mathcal{C}(x, y)=\sum_{n=0}^{\infty} \phi_{n}(x) \phi_{n}(y)
$$

Then simply $\mathcal{C} \phi_{n}(x)=\int \mathcal{C}(x, y) \phi_{n}(y) d y=(-1)^{n} \phi_{n}(x)$ (see, e.g., [42]). There are $\mathcal{P}^{2}=\mathcal{C}^{2}=\mathbb{I}$, but $\mathcal{P} \neq \mathcal{C}$, and $[\mathcal{P}, \mathcal{C}] \neq 0$ but $[\mathcal{C}, \mathcal{P} \mathcal{T}]=0$ and $[\mathcal{C}, H]=0$. The problem is that the calculation of $\mathcal{C}$ is very nontrivial for a given $H$ : One have to find solutions of the eigenvalue problem for this $H$. Having the $\mathcal{C}$ operator one can define observables.

In ordinary quantum mechanics the condition for a linear operator $A$ to be an observable is that $A$ has to be self-adjoint: $A=A^{+}$. This condition provides the expectation value $\langle\phi|A| \phi\rangle$ of $A$ in a given normalized state $\langle\phi \mid \phi\rangle=1$, to be real, Within the $\mathcal{P} \mathcal{T}$-symmetric quantum theory this condition is replaced by the following one: $A^{C P T} \stackrel{\text { def }}{=} \mathcal{C P} \mathcal{T} A \mathcal{C P} \mathcal{T}=A^{T}$, where $A^{T}$ denotes the transpose of $A$ [42]. This means that if $A$ satisfies this condition then the expectation value of $A$ calculated for a given state using $\mathcal{C P} \mathcal{T}$ inner product is real [42] and therefore this operator $A$ can be considered as the observable. Note that this condition depends on $\mathcal{C}$ and the form of $\mathcal{C}$ is determined by solutions of the eigenvalue problem for $H$. Hence the inner product $(., .)^{C P T}$ depends on the choice of $H$. So, in general it may happen that an linear operator $A$ satisfies the condition $\mathcal{C P} \mathcal{T} A \mathcal{C P} \mathcal{T}=A^{T}=$ for $H$ but it does not satisfy analogous condition for problem described by a Hamiltonian $H_{1} \neq H$ (assuming that $H$ and $H_{1}$ does not have common eigenfunctions). Every $\mathcal{C P}$-symmetric Hamiltonian $H$ satisfies the condition $\mathcal{C P} \mathcal{T} H \mathcal{C P} \mathcal{T}=H^{T}=H$, so the Hamiltonian $H$ is an observable. Now having observables and expectations values one can think about uncertainty relations. It turns out that in typical models considered in $\mathcal{P} \mathcal{T}$-symmetric quantum mechanics the position $x$ and momentum $p$ are not observables (sse, e.g., [42]). Simply in these models, e.g., the expectation value of $x$ in the ground state is a negative imaginary number as it was shown in [42]. Thus there is no position operator in $\mathcal{P} \mathcal{T}$-symmetric quantum mechanics [42]. This means that there is no a place for the Heisenberg uncertainty relation (2) in $\mathcal{P} \mathcal{T}$-symmetric quantum mechanics. So, the question arises: can the system be prepared in a state, in which the position and momentum are sharply defined in such cases? Of course, within the $\mathcal{P T}$ - symmetric quantum mechanics one can try to find two non commuting observables $A$ and $B$, that is, such linear operators that $\mathcal{C P} \mathcal{T} A \mathcal{C P} \mathcal{T}=A^{T}$ and $\mathcal{C P} \mathcal{T} B \mathcal{C P} \mathcal{T}=B^{T}$, and to derive a relation corresponding to the uncertainty relation (4), but such a relation can never be considered to be universally valid. It is because the operator $\mathcal{C}$, the inner product, $(., .)^{C P T}$, in the state space and thus geometry of this state space are determined by a given Hamiltonian $H$ for the problem considered. In conclusion one may wonder if it makes sense to ask about uncertainty relations in $\mathcal{P} \mathcal{T}$-symmetric quantum mechanics.

In the light of the consequences of the $\mathcal{P} \mathcal{T}$-symmetric quantum mechanics and of the fact that within the standard quantum mechanics uncertainty relations only results from the geometry of the state space, the question concerning uncertainty relations may arise: are they the intrinsic and inherent property of the quantum systems, or maybe, are they a result of our choice of the state 
space? Taking into account applications of the $\mathcal{P} \mathcal{T}$-symmetric Hamiltonians in quantum field theory, quantum optics, in condensed matter physics, etc., and the reported result in [46,47], where a violation of Heisenberg's "measurement-disturbance relationship" was observed, this question seems to be nontrivial and important.

\section{Discussion and Conclusions}

As it was mentioned in the Introduction, There is almost common belief that having a pair of non-commuting self-adjoint operators (observables) $A$ and $B$ one always finds that the product of standard deviations $\Delta_{\phi} A$ and $\Delta_{\phi} B$ calculated for them, (where $|\psi\rangle \in \mathcal{H}$ ), is always larger than some nonzero positive number: $\Delta_{\phi} A \cdot \Delta_{\phi} B \geq a>0$. In Sections 3-5 it was shown that such a belief may lead to confusions. As it was shown in Section 3, in 2-dimensional, or 3-dimensional state spaces there are many examples of self-adjoint matrices (operators) and vectors in state spaces such that the product of the standard deviations calculated for them is greater than or equal to zero. Similar cases can be found in n-dimensional state spaces. These observations seem to be highly non-trivial in the case of studying the properties of two-, three-, and n-level quantum systems, which have many applications and which are intensively studied in the context of applications, e.g., in the theory of quantum computers, and in another cases. Simply, the examples presented in Section 3 show that the uncertainty principle (4) may not work in many cases in n-level systems, although at first glance it seems it must work. This means that in order to avoid unpleasant surprises, when examining such systems and drawing general conclusions from them based on the uncertainty principle, one must carefully check each such case.

Similar observations concern also, e.g., systems having the space $L^{2}(\mathbb{R})$, or $L^{2}([a, b]) \subset L^{2}(\mathbb{R})$ as a state space. Examples of such a situations has been studied in Section 4. In this Section the attention was focused on the standard Heisenberg position-momentum uncertainty relation (2) for a quantum particle in the box with perfectly reflecting and impenetrable walls. The detailed description of this problem can be found, e.g., in [34,35,37-39] and this is why we do not analyze all the details and subtleties of this problem, but focus our attention on the momentum of the particle considered. Much more details concerning this momentum can be found, e.g., in [37] and also in the nice paper [36]. In Section 4.1 the "standard" case of a particle in the box has been considered, when the potential $V(x)$ is given by formula (19) with $a=0$ and $b=l$ and the state space is $L^{2}([0, l])$. Analyzing the position-momentum uncertainty relation in this case the family of self-adjoint adjoint extensions of the momentum operator $P_{x}^{\vartheta}$ (see (22) and (23)) was used to find the uncertainty relation. Unfortunately, a naive direct use of the relation (4) to find the suitable relation leads to paradoxical situation, where the left-hand side of the relation (4) is zero for eigenvectors of $P_{x}^{\vartheta},|\phi\rangle=\left|u_{n}^{\vartheta}(x)\right\rangle$, whereas, according to (5) the right hand side is non-zero. A more detailed analysis shows that the position operator $X$ removes for any $\vartheta$ vectors $\left|u_{n}^{\vartheta}(x)\right\rangle$ from the domain, $D\left(P_{x}^{\vartheta}\right)$, of the operator $P_{x}^{\vartheta}$, which means that the commutator $\left[X, P_{x}^{\vartheta}\right]$ does not exist in this case and therefore the right hand side of the inequality (4) does not exits. What is more, it appears that for any $\vartheta$ the position operator $X$ removes also from $D\left(P_{x}^{\vartheta}\right)$ all vectors such that $\phi(0) \neq 0$, which has a consequence that in the case of these vectors the commutator $\left[X, P_{x}^{\vartheta}\right]$ can not be calculated. As a result the position-momentum uncertainty relation can not be derived from (4) in the mentioned cases (see also [37]).

A slightly different picture one meets in the "symmetric" case of the particle in the box discussed in Section 4.2. Here the Hilbert space $L^{2}\left(\left[-\frac{l}{2}, \frac{l}{2}\right]\right) \subset L^{2}(\mathbb{R})$ is the state space and for $0 \leq \vartheta \equiv \alpha+\pi<2 \pi$ the position operator $X$ moves vectors $|\phi(x)\rangle \in D\left(\Pi_{x}^{\alpha}\right)$ from the domain of the self-adjoint extension $\Pi_{x}^{\alpha}$ of the momentum operator to the domain $D\left(\Pi_{x}^{\alpha+\pi}\right) \neq D\left(\Pi_{x}^{\alpha}\right)$ of the operator $\Pi_{x}^{\alpha+\pi}$. Unfortunately this means that for $\mid \phi(x) \in D\left(\Pi_{x}^{\alpha}\right)$ vectors $X|\phi(x)\rangle \in D\left(\Pi_{x}^{\alpha+\pi}\right) \neq D\left(\Pi_{x}^{\alpha}\right)$ and therefore the matrix element of the commutator $\left\langle\phi(x)\left|\left[\Pi_{x}^{\alpha}, X\right]\right| \phi(x)\right\rangle$ calculated for $|\phi(x)\rangle=\left|u_{n}^{\alpha}(x)\right\rangle$, (where $\left|u_{n}^{\alpha}(x)\right\rangle$ is an eigenvector of $\left.\Pi_{x}^{\alpha}\right)$ can not exist. So, as the result one can not calculate the unceratinty relation (4) for the position and momentum. 
It seems that the root cause of these inconsistencies and paradoxes is the quantization procedure for particles whose spatial motion is confined to a finite volume. The standard canonical quantization procedure leads to the correct results if conventional space-phase variables $p_{x}$, (momentum), and $x$, (position), can vary from $-\infty$ to $+\infty$ : $\left|p_{x}\right|<\infty,|x|<\infty$. As it is stated in [48]: conventional phase-space variables, such as $p$ and $q$, where $-\infty<p, q<+\infty$, with Poisson brackets $\{q, p\}=1$, are natural candidates to promote to basic quantum operators in the procedures that canonical quantization employs. Simply if the spatial motion of the particle is confined to a finite volume then we have the constrained system and the quantization procedure should take into account this fact and to be consistent with the constraints.

As it has been shown the paradox appearing in the "symmetric" case can be removed if to use the observation that from the classical point of view the particle in the box is the system with constraints and to use the modified position operator, $X_{M}$, (defined by (33) and (34)) consistent with these constraints and replacing the standard position operator $X$. The use of the operator $X_{M}$ changes the commutator (5) giving the results (35)-(37). Applying the modified position operator to calculate $\Delta_{\phi} X_{M}$ for $\phi(x) \in L^{2}\left(-\left[\frac{l}{2}, \frac{l}{2}\right]\right)$ and inserting the commutators (36) into the right hand side of the inequality (4) may remove the above described inconsistencies appearing in the "symmetric" case considered in Section 4.2. Simply using the modified position operator $X_{M}$ and the commutator (35)-(37) one finds that expected value $\left\langle\left[P_{x}, X\right]\right\rangle_{\phi}$ of the commutator $\left[P_{x}, X\right]$ equals zero for $\phi(x)=u_{n}^{\alpha}(x)$ and also for $\phi(x)$ fulfilling the condition (38) and that described below this formula. This commutator is nonzero for $\phi(x)$ satisfying conditions (39). Summarizing this part, it should be noted that the properties of the modified position operator $X_{M}$ defined in Section 4.2 and its implications are a proposal that requires further in-depth studies.

One more observation concerning the "symmetric" case. It appears the in this case the potential $V(x)$ is not only symmetric with respect to the origin of the coordinate system but also with respect to the combined transformations of the space reflection, $\mathcal{P}$ and the inversion of time, $\mathcal{T}$, which are defined by Equations (42) and (43). The potential $V(x)$, and also eigenfunctions, $u_{n}^{\vartheta}(x)$ of the operator $\Pi_{x}^{\vartheta}$ and the domain, $D\left(\Pi_{x}^{\vartheta}\right)$, are invariant under the $\mathcal{P} \mathcal{T}$ transformation, which may explain slight differences between "standard" and "symmetric" cases of the particle in the box.

In general, the problem of the particle in the infinite square well has not only a long tradition of illustrating quantum concepts but also it has important practical meaning. Full and accurate knowledge of the properties of the particle in the potential well is necessary to understand the properties of such systems as quantum dots, quantum traps and and related problems. A problem of a single slit diffraction experiment and the uncertainty relation of position and momentum in such a system, where the spatial dimension is one $\left(x \in\left[-\frac{\Delta x}{2}, \frac{\Delta x}{2}\right]\right.$ - see [49]) is an example of such related problems: In [49] the uncertainty relation was evaluated for a subset of functions with support in $\left[-\frac{\Delta x}{2}, \frac{\Delta x}{2}\right]$, which satisfy Dirichlet conditions at $x=-\frac{\Delta x}{2}$ and $\left.x=+\frac{\Delta x}{2}\right]$. An another related problem is a problem of the uncertainty principle for a particle localized in a compact domain $D \subset \mathbb{R}^{n}$ considered in [50], where the approach used in [49] was applied. In this context, the information on the behavior of a particle resulting from the uncertainty principles seems to be of key importance for a full understanding of all the effects occurring in such systems and as it has been shown in Section 4 it is very nontrivial problem (see also, e.g., [39]) and still needs further studies.

The detailed and rigorous mathematical analysis of the Heisenberg's relation (2) together with (4) shows that, e.g., for observables $A \stackrel{\text { def }}{=} X^{n}$ and $B \stackrel{\text { def }}{=} P^{m}$, (where $P=-i \hbar \frac{d}{d x}$ and $m, n \in \mathbb{N}$ ), using the so-called unitary dilation operator one can build from a normalized state $|\psi(x)\rangle \in L^{2}(\mathbb{R})$ such a function that the product of standard deviations of $X^{n}$ and $P^{m}$ calculated for this function can vanish (for details see, e.g., [20]). This suggest that relations (2) and (4) may not be good relations, strictly speaking that the product $\Delta_{\psi} A \cdot \Delta_{\psi} B$ may not be a good measure of the uncertainty. This is why in many papers were considered other relations between standard deviations $\Delta_{\psi} A$ and $\Delta_{\psi} B[14,15]$ having, e.g., a form of a sum of the squares of the standard deviations (see e.g., [14]): $\| \delta_{0} A|\psi\rangle \|^{2}+$ $\| \delta_{0} B|\psi\rangle \|^{2} \geq c_{0}>0$, where $c_{0}$ is a real number and $\delta_{0} A, \delta_{0} B$ denote a suitably rescaled deviations $\delta A$ 
and $\delta B$ to have the same dimension, or to be dimensionless. A simple example of such a relation can be found analyzing the case of Pauli matrices considered in Section 3: It is enough to take a sum of squares of $\Delta_{\phi} \sigma_{x}$ and $\Delta_{\phi} \sigma_{y}$. As it is seen, the inconsistencies of this type and others discussed in previous Sections are integrated into inequality (4). For this reason, attempts are being made to improve and refine the Heisenberg as well as Robertson and Schrodinger uncertainty relations (see, e.g., [14-17,20]).

From the analysis presented in Section 5 it follows that a status and role of the uncertainty relations (1), (2) and (4) in $\mathcal{P} \mathcal{T}$-symmetric quantum theory seems to be unclear. It is because the definition of the observable is determined by the choice of $\mathcal{P} \mathcal{T}$-symmetric Hamiltonian $H$. This means that, for example, if $A$ and $B$ are observables with respect to the inner product $(., .)^{C_{1} P T}$ defined by means of the eigenfunction of the $\mathcal{P} \mathcal{T}$-symmetric Hamiltonian $H_{1}$, from which the operator $\mathcal{C}_{1}$ is build, then they need not be observables with respect to the inner product $(., .)^{C_{2} P T}$ defined by eigenvectors of such $H_{2} \neq H_{1}$ that $H_{1}$ and $H_{2}$ have not common eigenfunctions. Hence the relation corresponding to the uncertainty relation (4) can not be considered as universally valid: The relations derived for $H_{1}$ need not hold within $\mathcal{P} \mathcal{T}$-symmetric quantum mechanics generated by the $\mathcal{P} \mathcal{T}$-symmetric Hamiltonian $H_{2} \neq H_{1}$. What is more, as it was stated in [42], in typical models considered within $\mathcal{P} \mathcal{T}$-symmetric quantum mechanics the position and momentum are not observables. This means that the standard position-momentum uncertainty relation (2) can not be derived in such cases. In conclusion: Within the $\mathcal{P} \mathcal{T}$-symmetric quantum mechanics the problem of relations corresponding to the uncertainty relation (4) is open and needs further studies.

Funding: This research received no external funding.

Acknowledgments: This work was supported by the program of the Polish Ministry of Science and Higher Education under the name "Regional Initiative of Excellence" in 2019-2022, Project No. 003/RID/2018/19; (Funding amount: 11936 596.10 PLN).

Conflicts of Interest: The author declares that there are no conflict of interest regarding the publication of this article and that all results presented in this article are the author's own results.

\section{References}

1. Heisenberg, W. Uber den anschaulichen Inhalt der quantentheoretischen Kinematik und Mechanik. Z. Phys. 1927, 43, 172-198. [CrossRef]

2. Heisenberg, W. The Physical Principles of the Quantum Theory; Dover Publications Inc.: Mineola, NY, USA, 1949.

3. Robertson, H.P. The Uncertainty Principle. Phys. Rev. 1929, 34, 163. [CrossRef]

4. Schrödinger, E. Zum Heisenberschen Unschäfprinzip. Sitzungsber. Preuss. Akad. Wiss. 1930, XIX, 296-303.

5. Schrödinger, E. About Heisenberg Uncertainty Relation. arXiv 1999, arXiv:quant-ph/9903100.

6. Messiah, A. Quantum Mechanics; North Holland Publ. Co.: Amsterdam, The Netherlands, 1962; Volume I.

7. Merzbacher, E. Quantum Mechanics, 3rd ed.; John Wiley \& Sons, Inc.: New York, NY, USA, 1998.

8. Griffiths, D.J. Introduction to Quantum Mechanics; Pretinence Hall Inc.: Upper Saddle River, NJ, USA, 1995.

9. Urbanowski, K. Remarks on the uncertainty relations. Mod. Phys. Lett. 2020, 35, 2050219. [CrossRef]

10. Werner, R.F.; Farrelly, T. Uncertainty from Heisenberg to Today. Found. Phys. 2019, 49, 460-491. [CrossRef]

11. Busch, P.; Heinonen, T.; Lahti, P. Heisenberg's uncertainty principle. Phys. Rep. 2007, 452, 155-176. [CrossRef]

12. Busch, P.; Lahti, P.; Werner, R.F. Measurement uncertainty relations. J. Math. Phys. 2014, 55, 042111. [CrossRef]

13. Teschl, G. Mathematical Methods in Quantum Mechanics (With Applications to Schrödinger Operators); Graduate Studies in Mathematics; American Mathematical Society: Providence, RI, USA, 2009; Volume 99.

14. Folland, G.B.; Sitaram, A. The uncertainty principle: A mathematical survey. J. Fourier Anal. Appl. 1997, 3, 207-238. [CrossRef]

15. Cowling, M.G.; Price, J.F. Bandwidth versus time concentration: The Heisenberg-Pauli-Weyl inequality. SIAM J. Math. Anal. 1984, 15, 151-165. [CrossRef]

16. Dou, Y.N.; Du, H.K. Generalizations of the Heisenberg and Schrödinger uncertainty relations. J. Math. Phys. 2013, 54, 103508. [CrossRef]

17. Park, Y.M. Improvement of uncertinty relations for mixed states. J. Math. Phys. 2005, 46, 042109. [CrossRef] 
18. Rodriguez, E.B.; Aguilar, L.M.A. Disturbance-Disturbance uncertainty relation: The statistical distinguishability of quantum states determines disturbance. Sci. Rep. 2018, 8, 4010. [CrossRef] [PubMed]

19. Ozawa, M. Physical content of Heisenberg's uncertainty relation: Limitation and reformulation. Phys. Lett. A 2003, 318, 21-29. [CrossRef]

20. Dias, N.C.; Prata, J.N. Uncertainty relations for a non-canonical phase-space noncommutative algebra. J. Phys. A Math. Theor. 2019, 52, 225203. [CrossRef]

21. Kennard, E.H. Zur quantenmechanik einfacher bewegungstypen. Z. Phys. 1927, 44, 326-352. [CrossRef]

22. Ozawa, M. Universally valid reformulation of the Heisenberg uncertainty principle on noise and disturbance in measurement. Phys. Rev. A 2003, 67, 042105. [CrossRef]

23. Renes, J.M.; Scholz, V.B.; Huber, S. Uncertainty relations: An operational approach to the error-disturbance tradeoff. Quantum 2017, 1, 20. [CrossRef]

24. Busch, P.; Lahti, P.; Werner, R.F. Proof of Heisenberg's Error-Disturbance Relation. Phys. Rev. Lett. 2013, 111, 160405. [CrossRef]

25. Busch, P.; Lahti, P.; Werner, R.F. Colloquium: Quantum root-mean-square error and measurement uncertainty relations. Rev. Mod. Phys. 2014, 86, 1261-1281. [CrossRef]

26. Branciard, C. Error-tradeoff and error-disturbance relations forincompatible quantum measurements. Proc. Natl. Acad. Sci. USA 2013, 110, 6742-6747. [CrossRef] [PubMed]

27. Werner, R.F. The uncertainty relation for joint measurement of postion and momentum. Quantum Inf. Comput. 2004, 4, 546-562.

28. Ozawa, M. Quantum Limits of Measurements and Uncertainty Principle. In Quantum Aspects of Optical Communications; Bendjaballah, C., Hirota, O., Reynaud, S., Eds.; Springer: Berlin/Heidelberg, Germany, 1991; pp. 3-17.

29. Furrer, F.; Berta, M.; Tomamichel, M.; Scholz, V.B.; Christandl, M. Position-momentum uncertainty relations in the presence of quantum memory. J. Math. Phys. 2014, 55, 122205. [CrossRef]

30. Dammeier, L.; Schwonnek, R.; Werner, R.F. Uncertainty relations for angular momentum. New J. Phys. 2015, 17, 093046. [CrossRef]

31. Coles, P.J.; Berta, M.; Tomamichel, M. Entropic uncertainty relations and their applications. Rev. Mod. Phys. 2017, 89, 015002. [CrossRef]

32. Thekkadath, G.S.; Hufnagel, F.; Lundeen, J.S. Determining complementary properties using weak-measurement: Uncertainty, predictability, and disturbance. New J. Phys. 2018, 20, 113034. [CrossRef]

33. Werner, R.F. Uncertainty relations for general phase spaces. Front. Phys. 2016, 11, 110305. [CrossRef]

34. Robinett, R.W. Quantum Mechanics: Classical Results, Modern Systems, and Visualized Examples, 2nd ed.; Oxford University Press: Oxford, UK, 2006.

35. Bonneau, G.; Faraut, J.; Valent, G. Self-adjoint extensions of operators and the teaching of quantum mechanics. Am. J. Phys. 2001, 69, 322-331. [CrossRef]

36. Garbaczewski, P.; Karwowski, W. Impenetrable barriers and canonical quantization. Am. J. Phys. 2004, 72, 924-933. [CrossRef]

37. Gitman, D.M.; Tyutin, I.V.; Voronov, B.L. Self-Adjoint Extensions in Quantum Mechanics: General Theory and Applications to Schrödinger and Dirac Equations with Singular Potentials; Springer: New York, NY, USA, 2012.

38. Belloni, M.; Robinett, R.W. The infinite well and Dirac delta function potentials as pedagogical, mathematical and physical models in quantum mechanics. Phys. Rep. 2014, 540, 25-122. [CrossRef]

39. Al-Hashimi, M.H.; Wiese, U.-J. From a particle in a box to the uncertainty relation in a quantum dot and to reflecting walls for relativistic fermions. Ann. Phys. 2012, 327, 1-28. [CrossRef]

40. Mermin, N.D. Hidden variables and the two theorerns of John Bell. Rev. Mod. Phys. 1993, 65, 803-815. [CrossRef]

41. Sen, D. The uncertainty relations in quantum mechanics. Curr. Sci. 2014, 107, 203-218.

42. Bender, C.M. Making Sense of Non-Hermitian Hamiltonians. Rep. Prog. Phys. 2007, 70, 947-1018. [CrossRef]

43. Bender, C.M. Calculation of the Hidden Symmetry Operator in $\mathcal{P} \mathcal{T}$-Symmetric Quantum Mechanics. J. Phys. Math. Gen. 2003, 36, 1973-1983. [CrossRef]

44. Wang, Q. Calculation of $\mathcal{C}$ operator in $\mathcal{P} \mathcal{T}$-Symmetric Quantum Mechanics. Proc. Inst. Math. Nas Ukr. 2004, 50 Pt 2, 986-992.

45. Mostafazadeh, A. Pseudo-Hermitian Representation of Quantum Mechanics. Int. J. Geom. Methods Mod. Phys. 2010, 7, 1191-1306. [CrossRef] 
46. Rozema, L.A.; Darabi, A.; Mahler, D.H.; Hayat, A.; Soudagar, Y.; Steinberg, A.M. Violation of Heisenberg's Measurement-Disturbance Relationship by Weak Measurements. Phys. Rev. Lett. 2012, 109, 100404. [CrossRef]

47. Brumfie, G. Quantum uncertainty not all in the measurement: A common interpretation of Heisenberg's uncertainty principle is proven false. Nature 2012. [CrossRef]

48. Klauder, J.R. The Favored Classical Variables to Promote to Quantum Operators. arXiv 2020, arXiv:2006.13283v1.

49. Schürmann, T.; Hoffmann, I. A closer look at the uncertainty relation of position and momentum. Found. Phys. 2009, 39, 958-963. [CrossRef]

50. Schürmann, T. The uncertainty principle in terms of isoperimetric in- equalities. Appl. Math. 2017, 8, 307-311. [CrossRef]

(C) 2020 by the author. Licensee MDPI, Basel, Switzerland. This article is an open access article distributed under the terms and conditions of the Creative Commons Attribution (CC BY) license (http://creativecommons.org/licenses/by/4.0/). 